\title{
Sensor Networks with Decentralized Binary Detection: Clustering and Lifetime
}

\author{
Gianluigi Ferrari and Marco Martalò \\ Information Engineering Department \\ University of Parma \\ I-43100, Parma, Italy \\ Email: gianluigi.ferrari@unipr.it, martalo@tlc.unipr.it
}

\begin{abstract}
In this paper, we analyze the lifetime of clustered sensor networks with decentralized binary detection under a physical layer quality of service $(\mathrm{QoS})$ constraint, given by the maximum tolerable probability of decision error at the access point (AP). In order to properly model the network behavior, we consider four different distributions (exponential, uniform, Rayleigh, and lognormal) for the single sensors' lifetime. We show the benefits, in terms of longer network lifetime, of adaptive reclustering. On the other hand, absence of reclustering leads to a shorter network lifetime, and we show the impact of various clustering configurations under different QoS conditions. Our results show that the organization of sensors in a few big clusters is the winning strategy to maximize the network lifetime.
\end{abstract}

\section{INTRODUCTION}

Distributed detection has been an active research field for a long time [1]. The increasing interest for sensor networks has spurred a significant scientific activity in distributed detection techniques [2]. In the last years, an increasing number of civilian applications have been developed, especially in environmental monitoring [3], [4].

Several communication-theoretic-oriented approaches have been proposed to study decentralized detection [5], but most of them are based on the assumption of ideal communication links between the sensors and the access point (AP). However, in a realistic communication scenario, these links are likely to be noisy [6]. In [7], the authors follow a Bayesian approach for the minimization of the probability of decision error. In [8], the presence of noisy communication links, modeled as binary symmetric channels (BSCs), is considered and a few techniques are proposed to make the system more robust against the noise.

The problem of sensor network lifetime has also been studied extensively. In [9], the authors provide upper bounds on the lifetime of a sensor network and show specific sensor network scenarios where the derived bounds can actually be achieved. In [10], the authors use the lifetime maximization as the main criterion for the design of sensor networks with data gathering. In [11], the sensor network lifetime is interpreted as the time after which the first sensor in the network disconnects and a mathematical derivation of it is provided. Finally, in [12] the authors consider a realistic network of sensors equipped with TinyOS, an event-based operating system for networked sensor motes. In this scenario, the network lifetime is evaluated as a function of the average distance of the sensors from the central data collector.

In this paper, we consider a scenario where sensors are clustered and there are local fusion centers (FCs) associated with the clusters. All sensors observe a common binary phenomenon. This can be considered as an accurate model for realistic scenarios where sensors may form groups, depending on how they are placed and on the environmental characteristics. Each of the local FCs makes a decision based on the data collected from its sensors, and sends its decision to the final AP, which makes the final decision [13]. In [13], it is shown that uniform clustering leads to minimum performance degradation, in terms of probability of decision error, with respect to the case without clustering. In this paper, we analyze the lifetime of the proposed sensor network scenario with uniform clustering, considering a quality of service (QoS) condition given by the maximum tolerable probability of decision error. The analysis is carried out in two cases: (i) ideal reclustering, where the sensors, after each single sensor death, reconfigure themselves in uniform clusters; and (ii) absence of reclustering, where the initial cluster configuration remains fixed, regardless of the sequence of sensors' deaths. We show that in the absence of reclustering, the longest lifetime is guaranteed by an initial configuration characterized by the presence of a few big clusters.

The structure of this paper is the following. In Section II, communication-theoretic preliminaries on sensor networks with decentralized binary detection are given. In Section III, we propose a simple approach to evaluate the network lifetime under a physical layer-oriented QoS condition. The implications of the obtained results are discussed in Section IV, and concluding remarks are given in Section $\mathrm{V}$.

\section{Communication-Theoretic Preliminaries}

We consider a network scenario where $N$ sensors observe a common binary phenomenon. They are clustered into $n_{\mathrm{c}}<N$ groups, and each of them can communicate with only one local FC. The FCs collect data from the sensors in the corresponding cluster and make local decisions on the status of the binary phenomenon. At this point, each local FC transmits its decision to the AP, which makes a final decision on the status of the phenomenon. 
The status of the common binary phenomenon under observation is characterized as follows:

$$
H= \begin{cases}H_{0} & \text { with probability } p_{0} \\ H_{1} & \text { with probability } 1-p_{0}\end{cases}
$$

where $p_{0} \triangleq P\left(H=H_{0}\right)$. The observed signal at the $i$-th sensor can be expressed as

$$
r_{i}=c_{\mathrm{E}}+n_{i} \quad i=1, \ldots, N
$$

where

$$
c_{\mathrm{E}} \triangleq \begin{cases}0 & \text { if } H=H_{0} \\ s & \text { if } H=H_{1}\end{cases}
$$

Assuming that the noise samples $\left\{n_{i}\right\}$ are independent with the same Gaussian distribution $\mathscr{N}\left(0, \sigma^{2}\right)$, the common signalto-noise ratio (SNR) at the sensors can be defined as follows:

$$
\mathrm{SNR}_{\text {sensor }}=\frac{\left[\mathrm{E}\left\{\mathrm{c}_{\mathrm{E}} \mid \mathrm{H}_{1}\right\}-\mathrm{E}\left\{\mathrm{c}_{\mathrm{E}} \mid \mathrm{H}_{0}\right\}\right]^{2}}{\sigma^{2}}=\frac{s^{2}}{\sigma^{2}} .
$$

Each sensor makes a decision comparing the observation $r_{i}$ with a threshold value $\tau_{i}$ and computes a local decision $u_{i}=$ $U\left(r_{i}-\tau_{i}\right)$, where $U(\cdot)$ is the unit step function. In order to optimize the system performance, the threshold $\tau_{i}$ at the $i$-th sensor needs to be properly chosen. In this paper, we use a common threshold value $\tau$ for all sensors but, in general, this strategy might not be the best. While in a scenario with no clustering and ideal communication links between the sensors and the AP the relation between $\tau$ and $s$ is well known [7], in the presence of clusters the decision threshold $\tau$ needs to be optimized in order to minimize the probability of decision error. We underline that this optimization is carried out in all results presented in the following.

In an ideal scenario, the $N$ sensors observe the common binary phenomenon $H$ and send their decisions $\left\{u_{i}\right\}$ to the $n_{\mathrm{c}}$ FCs. Each of the $n_{\mathrm{c}}$ clusters contains $d_{\mathrm{c}}$ sensors, with $N=$ $n_{\mathrm{c}} \cdot d_{\mathrm{c}}$. The $j$-th FC, $j=1, \ldots, n_{\mathrm{c}}$, performs an information fusion, and computes a local decision according the following majority-like rule [7]:

$$
\widehat{H}_{j}=\Gamma\left(u_{1}^{(j)}, \ldots, u_{d_{\mathrm{c}}}^{(j)}\right)= \begin{cases}0 & \text { if } \sum_{m=1}^{d_{\mathrm{c}}} u_{m}^{(j)}<k \\ 1 & \text { if } \sum_{m=1}^{d_{\mathrm{c}}} u_{m}^{(j)} \geq k\end{cases}
$$

where $k$ is the threshold at the FCs. ${ }^{1}$

The decisions generated by the FCs are sent to the AP, which makes the following final decision:

$$
\widehat{H}=\Theta\left(\widehat{H}_{1}, \ldots, \widehat{H}_{n_{\mathrm{c}}}\right)= \begin{cases}H_{0} & \text { if } \sum_{m=1}^{n_{\mathrm{c}}} \widehat{H}_{m}<k_{\mathrm{f}} \\ H_{1} & \text { if } \sum_{m=1}^{n_{\mathrm{c}}} \widehat{H}_{m} \geq k_{\mathrm{f}}\end{cases}
$$

where $k_{\mathrm{f}}$ is the AP threshold. Using a combinatorial approach (based on the use of repeated trials formula [14]), one can write the probability of decision error as [13]

$$
\begin{aligned}
& P_{\mathrm{e}}=p_{0} \operatorname{bin}\left(k_{\mathrm{f}}, n_{\mathrm{c}}, n_{\mathrm{c}}, i, \operatorname{bin}\left(k, d_{\mathrm{c}}, d_{\mathrm{c}}, j, 1-\Phi(\tau)\right)\right) \\
& \left.\quad+\left(1-p_{0}\right) \operatorname{bin}\left(0, k_{\mathrm{f}}-1, n_{\mathrm{c}}, i, \operatorname{bin}\left(k, d_{\mathrm{c}}, d_{\mathrm{c}}, j, 1-\Phi(\tau-s)\right)\right)\right)
\end{aligned}
$$

\footnotetext{
${ }^{1}$ The threshold $k$ is the same for all the FCs because the clusters are supposed to have the same dimension. An extension to the case of non-uniform clustering is provided in [13].
}

where $\Phi(x) \triangleq \int_{-\infty}^{x} \frac{1}{\sqrt{2 \pi}} \exp \left(-y^{2} / 2\right) \mathrm{d} y$ and $\operatorname{bin}(a, b, n, z) \triangleq$ $\sum_{i=a}^{b}\left(\begin{array}{c}n \\ i\end{array}\right) z^{i}(1-z)^{(n-i)}, 0 \leq z \leq 1$. It is possible to show that the probability of decision error (3) reduces to that derived in [8] if $n_{\mathrm{c}}=d_{\mathrm{c}}=1$, i.e., there is no clustering. The proposed approach can be straightforwardly extended to decentralized detection schemes with a generic number of decision levels, i.e., schemes characterized by the presence of more than one layer of FCs between the sensors and the AP [15].

In general, one can assume that the communication links are noisy. In [8], a noisy link is modeled as a BSC with crossover probability $p$. In particular, we assume noisy links only between the sensors and the FCs. The higher-level links in the network, i.e., those between the FCs and the AP, are assumed ideal. In fact, in a realistic scenario, the network designer is likely to be able to control the placement of the FCs in the environment to be monitored. Therefore, the links between FCs and AP can be considered more reliable. Note that in order to model a realistic wireless communication link (affected, for example, by Rayleigh fading), a BSC might not be the best choice. However, we point out that the simple framework proposed in this paper can be extended to account for more realistic communication constraints.

In order to extend the previous analytical approach to a scenario with noisy links, one can simply observe that only the terms $1-\Phi(\tau)$ and $1-\Phi(\tau-s)$ in (3) have to be properly modified, with respect to an ideal scenario, in order to take into account the presence of noise [8]. More precisely, these terms have to be replaced, respectively, by

$$
\begin{aligned}
& P_{\mathrm{c}_{0}} \triangleq[1-\Phi(\tau)](1-p)+\Phi(\tau) p \\
& P_{\mathrm{c}_{1}} \triangleq[1-\Phi(\tau-s)](1-p)+\Phi(\tau-s) p .
\end{aligned}
$$

More details can be found in [8]. In the following, we will investigate the network behavior only in the case of ideal communication links, since the focus of this paper is on the impact of clustering on the network lifetime. However, the extension of our results to noisy communication links is straightforward. In fact, the noise in the communication links causes a performance degradation, i.e., the probability of decision error becomes higher [8]. Therefore, the network lifetime will reduce since the QoS condition can be satisfied for a shorter period (as will be explained more clearly in Section III).

In Fig. 1, the probability of decision error is shown, as a function of the sensor SNR, in three possible scenarios with $N=16$ sensors: (i) absence of clustering; (ii) uniform clustering; and (iii) non-uniform clustering. Both analytical (lines) and simulation (symbols) results are shown. As one can observe, there is excellent agreement between them. For nonuniform clustering, the derivation of the probability of decision error is similar to that outlined in this section. However, since the dimensions of the clusters are different, the derivation of the probability of decision error requires the use of a generalized version of the repeated trials formula. All the topologies with uniform clustering, i.e., 8-8 (2 clusters with 8 sensors each), 4-4-4-4 (4 clusters with 4 sensors each), and 


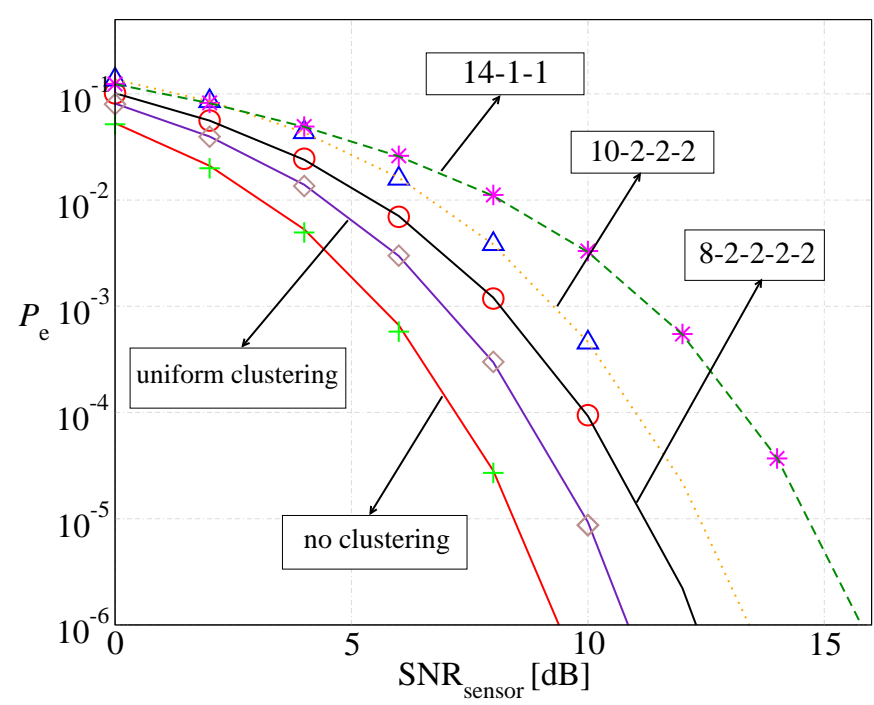

Fig. 1. Probability of decision error, as a function of the sensor SNR, in a scenario with $N=16$ sensors and equal a priori costs of the phenomenon ( $p_{0}=p_{1}=1 / 2$ ). Three different topologies are considered: (i) absence of clustering, (ii) uniform clustering, and (iii) non-uniform clustering (in this case, the specific configurations are indicated explicitly). Lines are associated with analytical results, whereas symbols are associated with simulation results.

2-2-2-2-2-2-2-2 (8 clusters with 2 sensors each), lead to the same performance curve. Therefore, one can conclude that the performance does not depend, as long as clustering is uniform, on the particular subdivision of the sensors among the clusters. For comparison, the curves associated with absence of clustering and with non-uniform clustering are also shown. It is clear that the higher is the non-uniformity degree, the worse is the performance. On the other hand, uniform clustering leads to the minimum loss with respect to the performance in the absence of clustering. This loss is the lowest possible. In the remainder of this paper, we will consider only scenarios with uniform clustering. Based on the following derivation and the results in Fig. 1, the reader can predict that the presence of non-uniform clustering will lead to a significant network lifetime reduction.

In Fig. 2, the probability of decision error is shown, as a function of the sensor SNR, for different values of the number of sensors $N$ in a scenario with uniform clustering and equal a priori costs of the phenomenon $\left(p_{0}=p_{1}=1 / 2\right)$. In particular, the considered values of $N$ are: (i) $N=16$, (ii) $N=20$, (iii) $N=32$, (iv) $N=40$, and (v) $N=64$. One can observe that only one curve is plotted for every value of $N$, as we have previously shown that the performance does not depend on the number of clusters (for a given $N$ ), as long as clustering is uniform. Obviously, the performance improves (i.e., the probability of decision error decreases) when the number of sensors in the network increases. The results in Fig. 2 will be used in Section III to compute the sensor network lifetime under a QoS condition on the maximum acceptable probability of decision error.

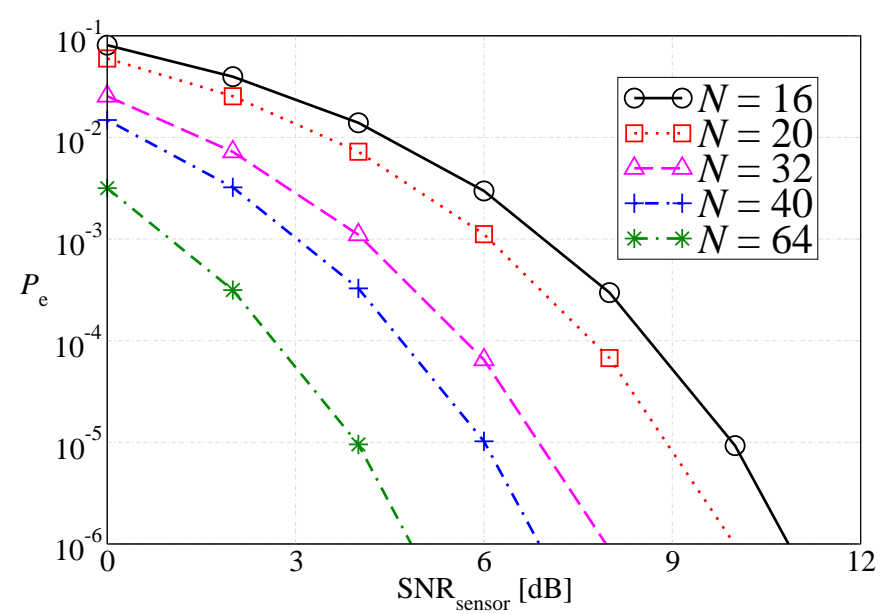

Fig. 2. Probability of decision error, as a function of the sensor SNR, in a scenario with uniform clustering and equal a priori costs of the common binary phenomenon $\left(p_{0}=p_{1}=1 / 2\right)$. Different values of the number of sensors are shown.

\section{Sensor Network LifETIME UNDER A PHYSiCAL LAYER QOS CONDITION}

In order to evaluate the sensor network lifetime, one needs first to define when the network has to be considered "alive." We assume that the network is "alive" until a given QoS condition is satisfied. Since the network performance is characterized in terms of probability of decision error, the chosen QoS condition is the following:

$$
P_{\mathrm{e}} \leq P_{\mathrm{e}}^{*}
$$

where $P_{\mathrm{e}}^{*}$ is the maximum tolerable probability of decision error. When a sensor in the network dies (e.g., its battery exhausts), the probability of decision error increases since a lower number of sensors is alive (see, for instance, Fig. 2). Moreover, the presence of a specific clustering configuration might accelerate the process of network death. More precisely, the network dies when the desired QoS condition (4) is no longer satisfied, as a consequence of the death of a critical sensor. Therefore, the network lifetime corresponds to the lifetime of this critical sensor. Obviously, the criticality of a sensor depends on the particular sequence of sensors' deaths.

Based on the considerations in the previous paragraph, in order to estimate the network lifetime one needs to consider a reasonable model for the sensor lifetime. We denote by $F(t)$ the cumulative density function (CDF) of the sensors' lifetime and we consider the following four distributions as 
representative for a sensor lifetime:

$\begin{array}{lll}\text { Exponential: } & F(t)=\left[1-e^{-t / \mu}\right] U(t) \\ \text { Uniform: } & F(t)=\left\{\begin{array}{cl}0 & \text { if } t<0 \\ \frac{t}{t_{\max }} & \text { if } 0 \leq t \leq t_{\max } \\ 1 & \text { if } t>t_{\max }\end{array}\right. \\ \text { Rayleigh: } & F(t)=\left[1-e^{\left.-t^{2} / 2 \sigma_{\text {ray }}^{2}\right] U(t)}\right. \\ \text { Lognormal: } & F(t)=\left[\frac{1}{2}+\frac{1}{2} \operatorname{Erf}\left(\frac{\log (t)-\zeta}{\sqrt{2 \sigma_{\log }^{2}}}\right)\right] U(t)\end{array}$

where $\operatorname{Erf}(x) \triangleq \frac{2}{\sqrt{\pi}} \int_{-\infty}^{x} \exp \left(-y^{2}\right) \mathrm{d} y$ is the error function and the time $t$ is measured in arbitrary units ([aU]). We have chosen the distributions in (5) as good models for the sensor lifetime. In fact, a realistic sensor should have a characteristic average value, whereas longer or shorter lifetimes should be less likely. Distributions like those proposed in (5), with the exception of the uniform distribution (which is, however, an interesting distribution), comply with these characteristics. In order to obtain a "fair" comparison between different sensor lifetime distributions, we impose that the average sensor lifetime is the same for all the proposed distributions in (5). Without loss of generality, we fix the average value of the exponential distribution, i.e., $\mu$, and we impose that the other lifetime distributions have the same average value. After a few manipulations, one obtains that the parameters in the remaining distributions in (5) need to be set as follows:

$$
\begin{aligned}
t_{\max } & =2 \mu \\
\sigma_{\text {ray }} & =\sqrt{\frac{2 \mu^{2}}{\pi}} \\
\zeta+\frac{\sigma_{\log }^{2}}{2} & =\log (\mu) .
\end{aligned}
$$

In particular, for a lognormal distribution (associated with the last condition in (6)) there are two free parameters: $\zeta$ and $\sigma_{\log }$. Therefore, one can set arbitrarily one of the two parameters, deriving the other parameter consequently. In the following, various possibilities will be considered.

\section{A. Analysis with Ideal Reclustering}

As mentioned in Section II, we are interested in analyzing the network behavior when the QoS condition (4) is satisfied. In this subsection, we consider the case of ideal reclustering, i.e., we assume that the network dynamically reconfigures its topology, immediately after a sensor death, in order to recreate a uniform configuration. The analysis presented in this paper does not take into account the time for reclusterization. This time depends on the specific strategy chosen in order to reconfigure correctly the connections between the sensors and the FCs, ${ }^{2}$ and between the FCs and the AP. We are currently investigating this aspect.

Given a maximum tolerable probability of decision error $P_{\mathrm{e}}^{*}$, one can determine the lowest number of sensors, denoted

\footnotetext{
${ }^{2}$ We point out that a FC could be an "enhanced" sensor.
}

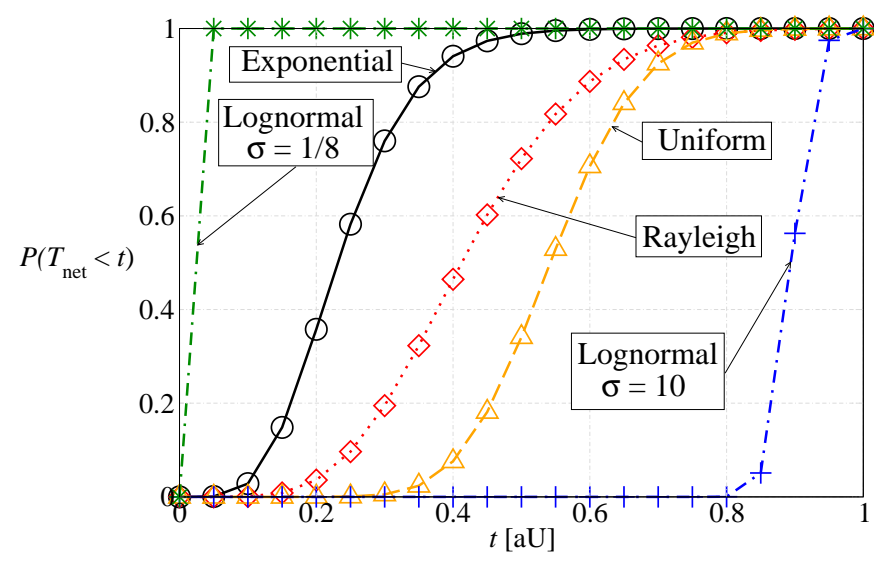

Fig. 3. CDF of the network lifetime, as a function of time, in a scenario with $N=32$ sensors, uniform clustering, ideal reclustering, and $\mathrm{SNR}_{\mathrm{sensor}}=5$ $\mathrm{dB}$. The QoS condition is set to $P_{\mathrm{e}}^{*}=10^{-3}$. All the distributions in (5) are considered. Lines are associated with analysis, whereas symbols are associated with simulations.

as $N^{\text {min }}$, required to satisfy the desired QoS condition. For instance, considering Fig. 2 and fixing a maximum tolerable value $P_{\mathrm{e}}^{*}$, one can observe that, for decreasing number of sensors, at some point the actual probability of decision error $P_{\mathrm{e}}$ becomes higher than $P_{\mathrm{e}}^{*}$. In other words, the probability of decision error is lower than $P_{\mathrm{e}}^{*}$ if at least $N^{\mathrm{min}}$ sensors are alive or, equivalently, until $N-N^{\mathrm{min}}+1$ sensors die. Therefore, denoting as $T_{\text {net }}$ the network lifetime, one can write:

$$
P\left(T_{\text {net }} \leq t\right)=P\left\{\begin{array}{l}
\text { at least } N-N^{\mathrm{min}}+1 \\
\text { sensors have } T_{\text {sensor }}<t
\end{array}\right\}
$$

where $T_{\text {sensor }}$ is the sensor lifetime (this random variable has the same distribution for all sensors). Since the sensors lifetimes are supposed independent, using the repeated trials formula, one obtains

$$
P\left(T_{\text {net }} \leq t\right)=\sum_{i=N-N^{\min }+1}^{N}\left(\begin{array}{c}
N \\
N-N^{\text {min }}+1
\end{array}\right) P_{\text {die }}^{i}\left(1-P_{\text {die }}\right)^{N-i}
$$

where $P_{\mathrm{die}} \triangleq P\left(T_{\text {sensor }} \leq t\right)$ is the probability that a sensor lifetime $T_{\text {sensor }}$ is lower than or equal to $t$, i.e., the CDF of $T_{\text {sensor }}$.

In Fig. 3, the CDF of the network lifetime is shown, as a function of time, in a scenario with $N=32$ sensors grouped in uniform clusters and in the presence of ideal reclusterization. The sensor SNR is set to $5 \mathrm{~dB}$ and the maximum tolerable probability of decision error is $P_{\mathrm{e}}^{*}=10^{-3}$. In particular, we fix the average value of the exponential distribution to $\mu=1$ and, consequently, we derive the values for the parameters of the other distributions according to (6), obtaining $t_{\max }=2$ (uniform distribution) and $\sigma_{\text {ray }}=0.8$ (Rayleigh distribution). For the lognormal distribution, instead, we use two possible values for $\sigma_{\log }$ (10 and $1 / 8$, respectively) and, consequently, two values for $\zeta(-50$ and -0.008 , respectively). We point out that a lognormal distribution allows to model, through proper choice of the parameters $\zeta$ and $\sigma_{\log }$, a large variety of realistic sensor lifetime distributions. In Fig. 3, both analytical (lines) 
and simulation (symbols) results are shown. As one can note, there is excellent agreement between them.

\section{B. Absence of Reclustering}

In Section III-A, we have analyzed the network evolution in an ideal scenario where the topology is dynamically reconfigured in response to a sensor failure (e.g., because of the depletion of its battery). While the previous scenario assumes ideal reclusterization, in general it might happen that the initial cluster configuration is fixed. In this case, the following question is relevant: is there an optimum initial topology which leads to longest network lifetime? In order to answer this question, we will analyze the network evolution in scenarios where there is no cluster reconfiguration. As in Section III-A, the network is considered dead when the QoS condition (4) is no longer satisfied.

In the absence of ideal reclustering, an analytical performance evaluation is not feasible, i.e., there does not exist a closed-form expression for the CDF of the network lifetime. In fact, the CDF depends on the particular network evolution, i.e., it depends on how the sensors die among the clusters in the network. Therefore, each sequence of sensors' deaths is characterized by a specific lifetime, and one needs to resort to simulations in order to extrapolate an average statistical characterization. The simulations are performed according to the following steps:

1. generate the lifetimes of all $N$ sensors according to the chosen distribution and assign randomly the sensors to the clusters;

2. order the sensors' lifetimes for increasing values;

3. update the network topology after a sensor death;

4. compute the probability of decision error corresponding to the surviving topology determined at the previous point: if the QoS condition (4) is satisfied, then go back to 3 ., otherwise go to 5 .;

5. the lifetime of the last sensor which died is the network lifetime.

In Fig. 4, the CDF of the network lifetime is shown, as a function of time, in a scenario with $N=32$ sensors grouped, respectively, in 2, 4, and 8 clusters. The sensor SNR is set at $5 \mathrm{~dB}$ and the maximum tolerable probability of decision error is $P_{\mathrm{e}}^{*}=10^{-3}$. The sensors' lifetimes are modeled with an exponential distribution (similar considerations can be carried out for the other distributions in (5)). For comparison, the curve associated with ideal reclustering is also shown. One can observe that the higher is the number of clusters, the worse is the performance, i.e., the higher is the probability of network death. Moreover, the curve associated with 2 clusters is very close to that relative to ideal reclustering. In fact, in a scenario with only 2 clusters, the average number of sensors which die in each cluster is approximately the same and, consequently, the topology remains approximatively uniform. ${ }^{3}$

\footnotetext{
${ }^{3}$ From the results in Fig. 4, it seems that the scenario with absence of reclustering and 2 clusters is better than that with ideal reclustering. However, this behavior is due to the fact that the simulations were not sufficiently long. Longer simulations are currently running to generate more accurate results.
}

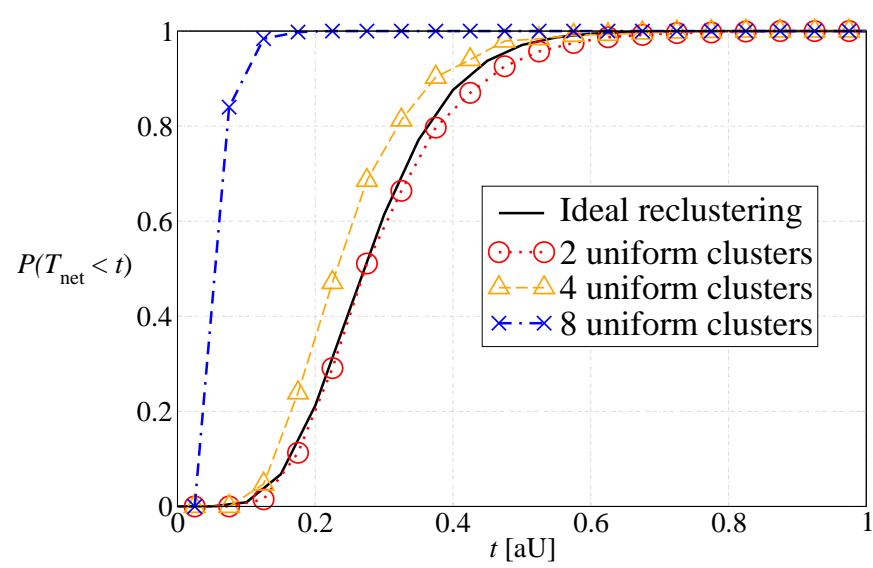

Fig. 4. CDF of the network lifetime, as a function of time, in a scenario with $N=32$ sensors, uniform clustering (with, respectively, 2, 4, and 8 clusters), and absence of reclustering. The sensor SNR is set to $5 \mathrm{~dB}$ and the maximum tolerable probability of decision error is $P_{\mathrm{e}}^{*}=10^{-3}$. For comparison, the curve relative to ideal reclustering is also shown. The distribution of the sensors' lifetime is exponential.

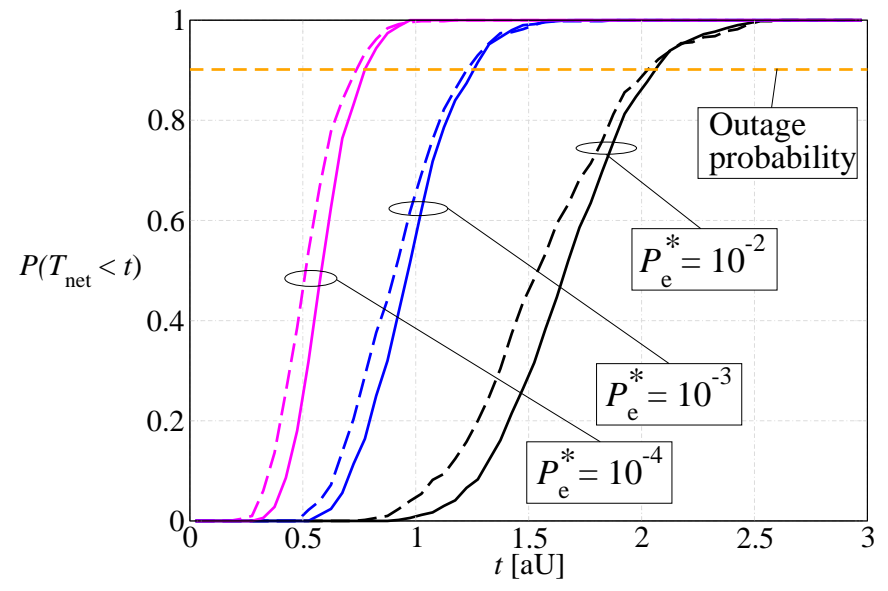

Fig. 5. CDF of the network lifetime, as a function of time, in a scenario with $N=64$ sensors, $\mathrm{SNR}_{\text {sensor }}=5 \mathrm{~dB}$, and absence of reclustering. Three values for the maximum tolerable probability of decision error are considered: (i) $P_{\mathrm{e}}^{*}=10^{-2}$, (ii) $P_{\mathrm{e}}^{*}=10^{-3}$, and (iii) $P_{\mathrm{e}}^{*}=10^{-4}$. Solid lines correspond to an initial topology with 2 clusters, whereas dashed lines are associated with an initial topology formed by 4 clusters.

In Fig. 5, the CDF of the network lifetime is shown, as a function of time, in a scenario with $N=64$ sensors, uniform clustering, and considering, respectively, 2 clusters (solid lines) and 4 clusters (dashed lines). The operating conditions are the same as in Fig. 4, and we consider three values for the maximum tolerable probability of decision error: $P_{\mathrm{e}}^{*}=10^{-2}$, $P_{\mathrm{e}}^{*}=10^{-3}$, and $P_{\mathrm{e}}^{*}=10^{-4}$, respectively. One can observe that, similarly to Fig. 4 , the higher is the number of clusters in the network, the shorter is the network lifetime. Moreover, the more stringent is the QoS condition (i.e., the lower is $P_{\mathrm{e}}^{*}$ ), the lower is the network lifetime. This is to be expected, since if $P_{\mathrm{e}}^{*}$ is very low, then a relatively small number of sensors need to die in order to make the entire network die.

\section{DISCUSSION}

In Table I, the network lifetime corresponding to a CDF equal to 0.9 (i.e., to a probability of outage of $90 \%$ ) is shown, 
TABLE I

NETWORK LIFETIME FOR OUTAGE PROBABILITY EQUAL TO 90\% IN A SCENARIO WITH $N=64$ NODES AND SNR sensor $=5$ DB. THE QOS CONDITIONS IN FIG. 5 ARE CONSIDERED.

\begin{tabular}{|c|c|c|c|c|}
\hline$P_{\mathrm{e}}^{*}$ & $\begin{array}{c}\text { Ideal } \\
\text { reclustering }\end{array}$ & $\begin{array}{c}\text { No reclustering } \\
(2 \text { clusters })\end{array}$ & $\begin{array}{c}\text { No reclustering } \\
(4 \text { clusters })\end{array}$ & $\begin{array}{c}\text { No reclustering } \\
(8 \text { clusters })\end{array}$ \\
\hline \hline $10^{-2}$ & 2.1 & 2.1 & 2.0 & 1.68 \\
\hline $10^{-3}$ & 1.3 & 1.3 & 1.2 & 1.012 \\
\hline $10^{-4}$ & 0.78 & 0.78 & 0.74 & 0.625 \\
\hline
\end{tabular}

assuming an exponential sensor lifetime, for various clustering configurations and various values of the maximum tolerable probability of decision error. The number of sensors is $N=64$. For comparison, the network lifetime with ideal reclustering is also shown. From the results in Table I, the following observations can be carried out.

- For a low number of clusters ( 2 or 4 ), the lifetime reduction, with respect to a scenario with ideal reclustering, is negligible. This is to be expected from the results in Fig. 4 and Fig. 5, and is due to the fact that the sensors die more or less uniformly in all clusters. When the number of clusters increases beyond 4 , the network lifetime starts reducing appreciably. Therefore, our results show that in the absence of ideal reclustering the winning strategy to prolong the network lifetime is to form a few large clusters.

- The impact of the QoS condition is very strong. In fact, when the QoS condition becomes more stringent (i.e, $P_{\mathrm{e}}^{*}$ decreases), the network lifetime decreases, since a lower number of sensor deaths is sufficient to violate this condition. On the other hand, if the constraint is less stringent, then a larger number of sensors have to die in order to violate the QoS condition.

- The impact of the number of nodes on the network lifetime has not been directly analyzed in this paper. However, since the performance improves when the number of sensors increases (as shown in Fig. 2), one can conclude that, for a fixed QoS condition, a network with a larger number of sensors will satisfy the QoS condition for a longer time and, therefore, the network lifetime will increase. Equivalently, one can impose a stronger QoS condition (a lower value of $P_{\mathrm{e}}^{*}$ ), still guaranteeing the same network lifetime.

\section{CONCLUding REMARKS}

In this paper, we have presented a simple framework to analyze the network lifetime in a clustered sensor network subject to a physical layer-oriented QoS condition, given by the maximum tolerable probability of decision error at the AP. First, we have derived a model for the sensor lifetime using a few distributions which are representative for a realistic sensor network. In the presence of ideal reclustering, the network lifetime is the longest possible. On the other hand, in the presence of a fixed clustered configuration, our results show that the number of clusters has a strong impact on the network lifetime. More precisely, the network lifetime is maximized if there are a few large clusters (at most 4). In all cases, the QoS condition has a strong impact on the network lifetime: the more stringent this condition is, the shorter is the network lifetime. It remains to be investigated what is the "cost" (in terms of energy consumption and delay) of the use of reclustering techniques.

\section{REFERENCES}

[1] J. N. Tsitsiklis, Adv. Statist. Signal Process., vol. 2, chapter Decentralized detection, pp. 297-344, Greenwich, CT: JAI Press, 1993, Eds.: H. V. Poor and J. B. Thomas.

[2] A. R. Reibman and L. W. Nolte, "Detection with distributed sensors," IEEE Trans. Aerosp. Electron. Syst., pp. 501-510, December 1981.

[3] C. Y. Chong and S. P. Kumar, "Sensor Networks: Evolution, Challanges, and Opportunities," Proc. IEEE, vol. 91, no. 8, pp. 1247-1256, August 2003.

[4] S. N. Simic and S. Sastry, "Distributed environmental monitorning using random sensor networks," in Proc. 2-nd Int. Work. on Inform. Processing in Sensor Networks, Palo Alto, CA, USA, April 2003, pp. 582-592.

[5] R. Viswanathan and P. K. Varshney, "Distributed detection with multiple sensors-Part I: Fundamentals," Proc. IEEE, vol. 85, no. 1, pp. 54-63, January 1997.

[6] T. S. Rappaport, Wireless Communications. Principles \& Pratice, 2nd Edition., Prentice-Hall, Uper Saddle River, NJ, USA, 2002.

[7] W. Shi, T. W. Sun, and R. D. Wesel, "Quasi-convexity and optimal binary fusion for distributed detection with identical sensors in generalized gaussian noise," IEEE Trans. Inform. Theory, vol. 47, no. 1, pp. 446-450, January 2001.

[8] G. Ferrari and R. Pagliari, "Decentralized detection in sensor networks with noisy communication links," in Proc. Tyrrhenian Int. Workshop on Digital Communications (TIWDC'05), Sorrento, Italy, July 2005.

[9] M. Bhardwaj, T. Garnett, and A. P. Chandrakasan, "Upper bounds on the lifetime of sensor networks," in Proc. IEEE International Conf. on Commun. (ICC), Amsterdam, the Netherlands, June 2001, vol. 119, pp. 785-790.

[10] K. Kalpakis, K. Dasgupta, and P. Namjoshi, "Maximum lifetime data gathering and aggregation in wireless sensor networks," Tech. Rep., University of Maryland, Baltimore, 2002, available at www. csee.umbc.edu/ kalpakis/.

[11] V. Rai and R. N. Mahapatra, "Lifetime modeling of a sensor network," in Proc. Design, Automation and Test in Europe 2006 (DATE'05), Messe Munich, Germany, March 2003, vol. 1, pp. 202-203.

[12] M. Ergen S. Coleri and T. J. Koo, "Lifetime analysis of a sensor network with hybrid automata modelling," in Proc. First Int. Workshop on Wireless Sensor Networks and Applications 2002 (WSNA'02), Atlanta, USA, September 2002, pp. 98-104.

[13] G. Ferrari, M. Martalò, and R. Pagliari, "Clustered decentralized binary detection: an information-theoretic approach," in Proc. Int. Symposium on Communications, Control and Signal Processing (ISCCSP'06), Marrakech, Morocco, March 2006.

[14] A. Papoulis, Probability, Random Variables and Stochastic Processes, McGraw-Hill, New York, NY, USA, 1991.

[15] G. Ferrari, M. Martalò, and R. Pagliari, "On multi-level decentralized binary detection in sensor networks," in Proc. Int. Conference on Intelligent Systems And Computing: Theory And Applications (ISYC'06), Ayia Napa, Cyprus, July 2006. 